\title{
Massive Proximal Extravasation as a Complication during Arthroscopic Anterior Cruciate Ligament Reconstruction
}

\author{
Etienne Cavaignac, MD, Regis Pailhé, MD, Nicolas Reina, MD, Philippe Chiron, MD, and Jean-Michel Laffosse, \\ $\mathrm{MD}$
}

Institut Locomoteur - Department of Orthopedic and Trauma Surgery, Rangueil University Center, Toulouse, France

\begin{abstract}
Extra-articular extravasation of irrigation fluid is relatively common around entry incisions and is usually limited to the subcutaneous tissue. Very rarely, extravasation occurs above the knee, in the thigh and even up into the pelvis. We are reporting the second case of irrigation fluid extravasation during a knee arthroscopy, which spread up to the thigh, groin and perineum during anterior cruciate ligament reconstruction, whilst the tourniquet was inflated. In our case, we think that the extravasation was caused by a fault in the pressure sensor due to the fact that the reservoir was over-filled. The irrigation pressure was therefore too high, and the irrigation fluid was able to diffuse, despite the presence of a pneumatic tourniquet, up past the thigh.
\end{abstract}

Keywords: Knee, Aneterior cruciate ligament, Complications

Complications rates during knee arthroscopy are low, between $1.4 \%$ and $1.68 \%{ }^{1,2)}$. Extra-articular extravasation of irrigation fluid is relatively common and is almost always limited to the subcutaneous tissue surrounding entry incisions ${ }^{3,4}$. There are no functional consequences and reabsorption is rapid. The fluid may leak into the thigh, popliteal fossa or the $\mathrm{leg}^{2}$. The extravasation of irrigation fluid above the knee joint into the thigh is rare ${ }^{2,3)}$. In the literature, only four cases have been reported, and in only one of these did the fluid reach the pelvis. A capsular tear was suspected as a reason of the extravasation in their report ${ }^{3}$. This report describes another case of extravasation above the knee and

Received December 16, 2012; Revised (1st) February 22, 2013;

(2nd) April 11, 2013; Accepted April 12, 2013

Correspondence to: Etienne Cavaignac, MD

Institut Locomoteur - Service de Chirurgie Orthopédique et

Traumatologique, CHU Rangueil, 1, Avenue Jean Poulhès TSA 50032,

31059 Toulouse Cedex 9, France

Tel: +33-6007342595, Fax: +33-561322232

E-mail: cavaignac.etienne@gmail.com

This is an Open Access article distributed under the terms of the Creative Commons Attribution Non-Commercial License (http://creativecommons.org/licenses/by-nc/3.0/) which permits unrestricted non-commercial use, distribution, and reproduction in any medium, provided the original work is properly cited. up into the pelvis during arthroscopic anterior cruciate ligament (ACL) reconstruction.

\section{Case Report}

A 35-year-old patient, a former amateur football player, was referred to us due to episodes of incapacitating instability of the right-knee, both at work and leisure. Ten years previously, he had suffered a femoral shaft fracture that was treated by intramedullary nailing, followed by removal of the nail 2 years later. Clinical examination and magnetic resonance imaging confirmed an old rupture of the ACL and a tear in the posterior horn of the medial meniscus.

We suggested an ACL reconstruction with the patellar bonetendon-bone graft. The operation was carried out under general anesthesia. The thigh was held in a clamp, with a pneumatic tourniquet (pressure, $250 \mathrm{mmHg}$ ) at the top of the leg. The arthroscopy pump used was the FMS DUO+ model (Depuy MITEK Inc., Raynham, MA, USA) which controls flow and pressure independently. The irrigation fluid used was a Lactated Ringer's isotonic solution with maximum pressure set to 100 mmHg. Exploration of the joint confirmed the ACL rupture as well the meniscal tear. The meniscus was minimally resected 
and the notch was cleaned. The patellar bone tendon bone was then taken. Tunnels were made independently from outside to inside. First the tibial tunnel (diameter $10 \mathrm{~mm}$ ) was drilled with an outside-in guide set at $50^{\circ}$. Secondly the outside-in femoral tunnel was drilled (diameter $9 \mathrm{~mm}$ ). A curet and a shaver were used to facilitate passage of graft into the tunnels. When the graft was passed through the femoral tunnel, one of the bone part broke. The graft was pulled back though but was lacerated and no longer usable, the broken bone part was not big enough to provide a good fixation. The hamstring tendons were not found during surgery because their dissection was compromised by the extravasation. As the patient had not been informed that

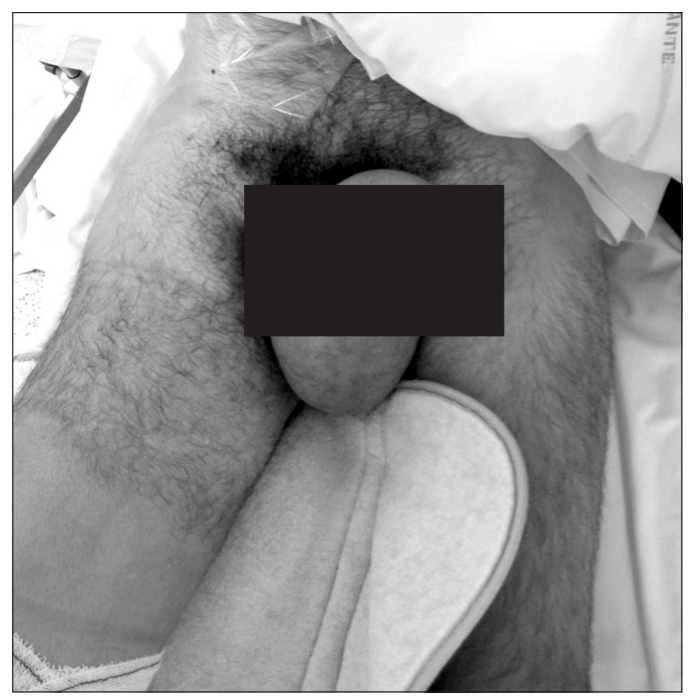

Fig. 1. Clinical appearance of the infiltration of the thigh, pelvis and scrotum due to extravasation of irrigation fluid.

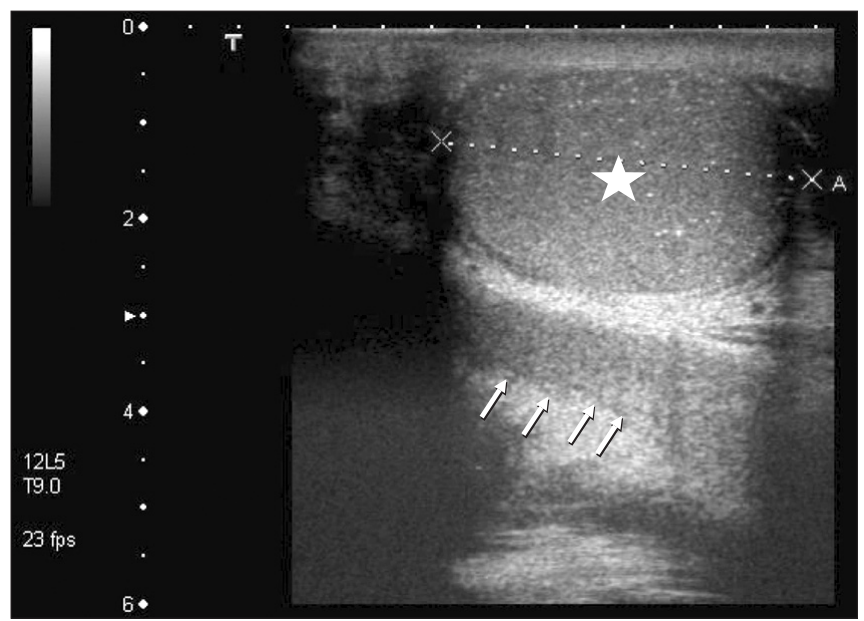

Fig. 2. Ultrasound view of the scrotum showing an effusion (white arrow) around a normal testicle (white star). a graft might be taken from the quadriceps tendon or from the other leg, the operation was abandoned after 105 minutes tourniquet time and around 15 liters of irrigation fluid. After removal of the drapes and tourniquet, swelling of the thigh, right iliac fossa, perineum and scrotum was noted (Fig. 1). There was no compartment syndrome and pulses (posterior tibial and dorsal pedis arteries) were all normal. When the patient awoke, there was no sensory or motor disturbance. Blood pressure was normal and there was no sign of blood loss. The diagnosis of irrigation fluid extravasation was immediately confirmed by an abdominal and pelvic ultrasound, which showed an effusion of the subperitoneal space and scrotum (Fig. 2).

Immediately after the surgery, we realized that the arthroscopy

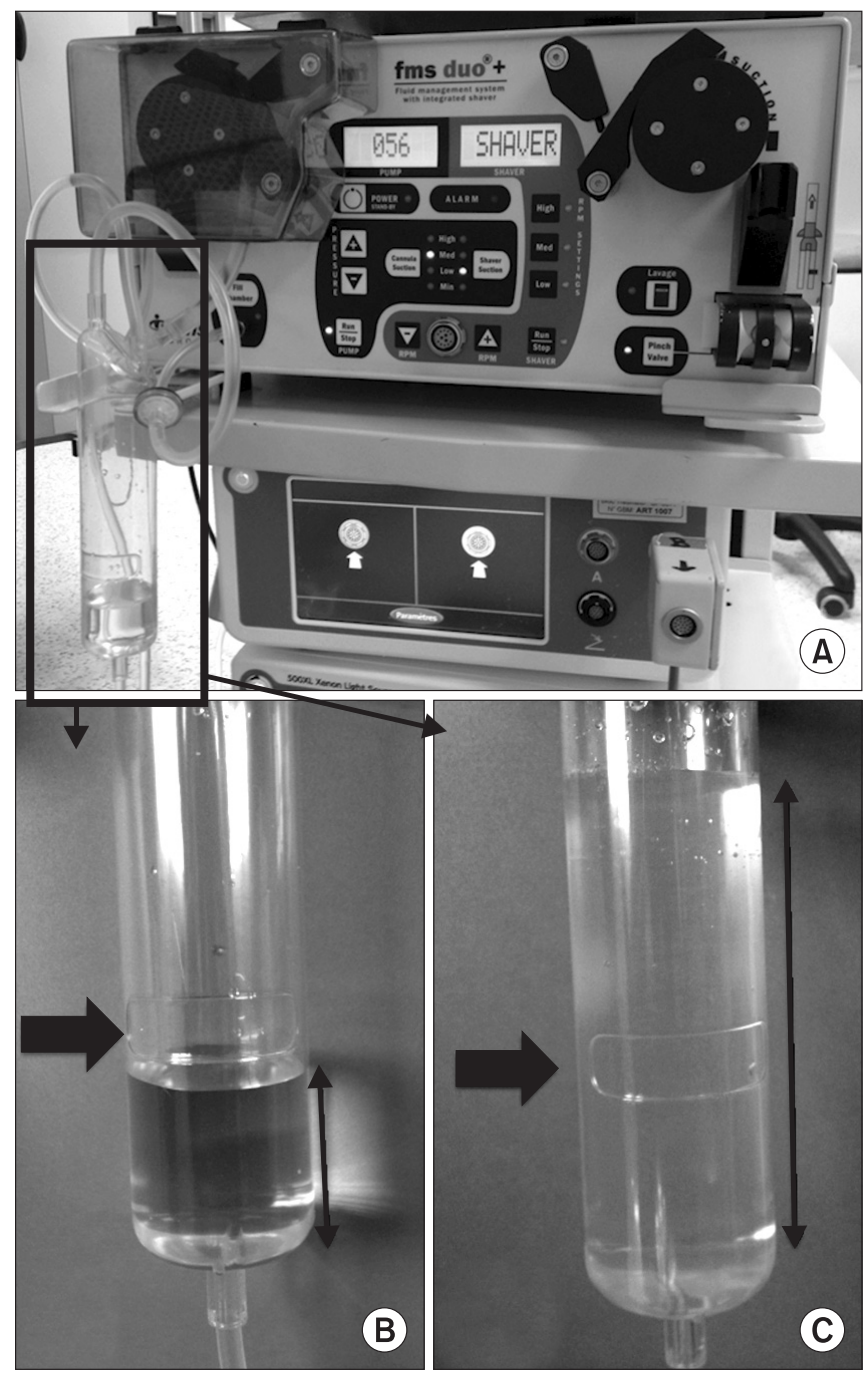

Fig. 3. View of the front of the arthroscopy pump and the reservoir (A). The reservoir is half-full (arrow), the pressure values are correct (B). The reservoir is completely filled (arrow), the pressure values are distorted (C). 

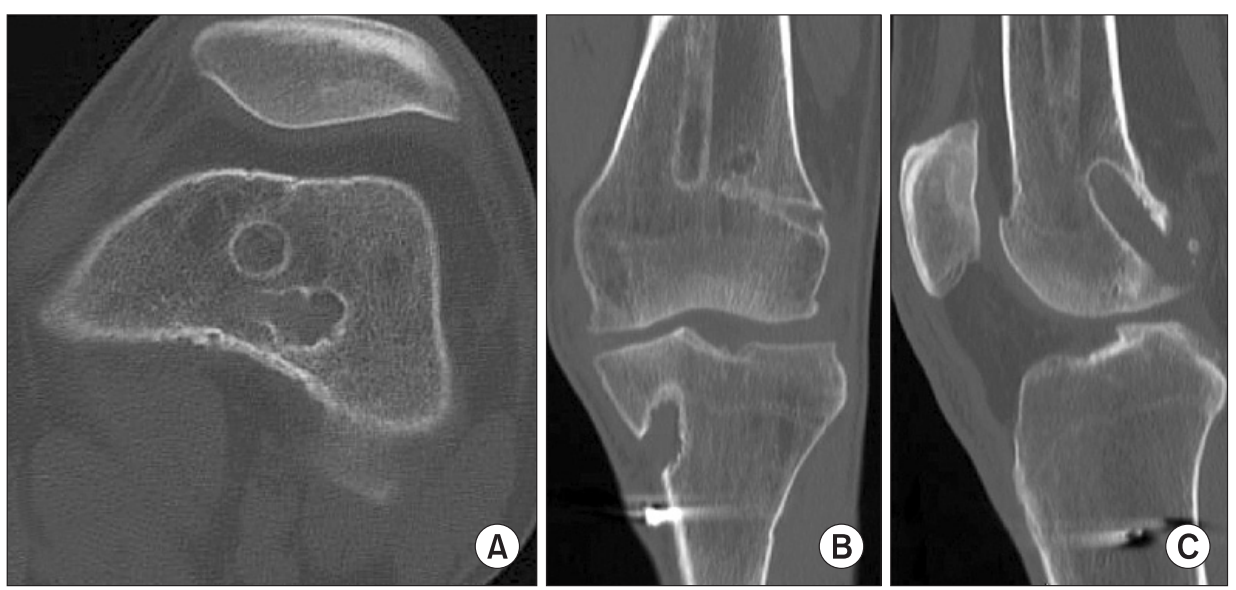

Fig. 4. Computed tomography (CT) image of the lower end of the femur showing the proximity of the femoral tunnel and the intramedullary cavity (nail position). (A) CT image cross-section. (B) Frontal reconstructed CT image. (C) Sagittal reconstructed CT image.

pump reservoir that would be normally half-filled was filled to maximum capacity (Fig. 3); the pressure values during the operation were much higher than those displayed. A urinary catheter was inserted to monitor diuresis, and the patient was treated with loop diuretics (furosemide) for 5 days. During the first two days, the furosemide dosage was $40 \mathrm{mg}$ twice a day; during the last 3 days, the dosage was $40 \mathrm{mg}$ a day. Electrolytes and diuresis were controlled every day in order to adapt the treatment. The urinary catheter was withdrawn at the end of the 5th day. A favorable outcome was rapidly achieved, without sequelae. Five months after this incident, we completed an ACL reconstruction using the quadriceps tendon, using the tunnels from the first surgery. Prior to doing this, we performed a computerized tomography scan to analyze the bone stock, which showed the proximity of the previous intramedullary nail position to the femoral tunnel (Fig. 4). At the last recall the patient presented with a stable and pain-free knee.

\section{Discussion}

The primary risk factors for extravasation of irrigation fluid into the thigh or calf after a knee arthroscopy are prolonged operation time, excessive intra-articular pressure and capsular tears ${ }^{5,6}$. Extravasation occurs most frequently below the knee, with a risk of compartment syndrome ${ }^{7,8)}$.

Several cases of extravasation above the knee are described in the literature. Bomberg et al. ${ }^{2)}$ reported 3 cases out of 283 arthroscopies. In these cases, the pressure delivered by the arthroscopy pump was $30 \mathrm{mmHg}$ and the tourniquet was never inflated. In 2 of these cases, they reported capsular tears that allowed fluid to escape. Romero et al. ${ }^{3)}$ reported a case of fluid extravasation above the knee with intraperitoneal accumulation of fluid after an arthroscopic meniscectomy. The pressure was set to $100 \mathrm{mmHg}$ and the tourniquet was not inflated. No capsular breach was found. In virtually all of these reported cases, there was a leak of fluid from the capsule with diffusion of the liquid either along the femoral artery where it is in contact with the vastus intermedius, or along the semimembranosus when the tourniquet was not inflated ${ }^{2,3)}$. The tourniquet may prevent prevent the diffusion of the liquid. The fall in pressure caused by the leakage was compensated by the arthroscopy pump, which had to deliver a larger volume of fluid in order to maintain sufficient pressure. Pressure sensors protect from intra-articular hypertension ${ }^{9)}$ but if the level of liquid in the sensor exceeds the operating limit as in the case presented here (Fig. 3) or in the event of a fault in the sensor itself, the pressure in the knee is no longer controlled and becomes too high. In a cadaver study, Noyes and Spievack ${ }^{4)}$ showed that a rupture of the suprapatellar pouch occurs when the pressures exceeds $200 \mathrm{mmHg}$ and the knee is flexed to $90^{\circ}$. Even in the absence of an obvious capsular breach, other warning signs should be heeded, in particular if the volume of irrigation fluid is too high and exceeds 15 liters ${ }^{10}$. In cases of prolonged surgery, therefore, a regular note should be taken into the inflows and outflows, so that any fluid leakage during the operation can be identified. Likewise, regular palpation of the compartments should be carried out due to the seriousness of compartment syndrome in the leg. Finally, the right choice of equipment, correct use of the arthroscopy pump and regular maintenance of the pressure sensors should prevent this type of complication ${ }^{9)}$. Concerning the treatment of this complication, the most important think is a careful observation of the patient to search signs of a compartmental syndrome. Without signs of compartmental syndrome, a conservative treatment is indicated ${ }^{3)}$.

In our case, we believe the over-filled reservoir led to a dysfunction of the pressure sensor which caused the extravasation. For 
us the lack of pressure control is the predisposing factor to this kind of complication. Concerning the diffusion of the fluid, we believe that two explanations are possible. 1) Fluid escaped along the femur, despite the presence of a pneumatic tourniquet, due to the narrow space between the femoral tunnel drilled for the ligament reconstruction and the empty medullary channel (from previous intramedullary nailing) (Fig. 4). The fluid rose into the intramedullary cavity as far as the trochanteric region before diffusing into the thigh and pelvis. 2) Due to the outsidein femoral technique tunnel, the fluid could spread into the soft tissue along the femoral artery (like in Romero et al.'s case $\left.{ }^{3}\right)$. The pneumatic tourniquet was not efficient enough (considering the high pressure of the irrigation fluid) to stop the phenomenon.

Extravasation of fluid upwards from the knee remains a rare complication of knee arthroscopy. It is more disturbing than dangerous, as it has always healed without sequelae, in our case as well as those described in the literature ${ }^{2,3)}$ due to the large capacity of the thigh and pelvis. This type of complication can be prevented by the use of an arthroscopy pump with a pressure sensor, regular testing of pressure sensors, and a record of inflows and outflows during long procedures.

\section{Conflict of Interest}

No potential conflict of interest relevant to this article was reported.

\section{References}

1. Small NC. Complications in arthroscopic surgery performed by experienced arthroscopists. Arthroscopy. 1988;4:215-21.

2. Bomberg BC, Hurley PE, Clark CA, McLaughlin CS. Complications associated with the use of an infusion pump during knee arthroscopy. Arthroscopy. 1992;8:224-8.

3. Romero J, Smit CM, Zanetti M. Massive intraperitoneal and extraperitoneal accumulation of irrigation fluid as a complication during knee arthroscopy. Arthroscopy. 1998;14:401-4.

4. Noyes FR, Spievack ES. Extraarticular fluid dissection in tissues during arthroscopy: a report of clinical cases and a study of intraarticular and thigh pressures in cadavers. Am J Sports Med. 1982;10:346-51.

5. Fowler J, Owens BD. Abdominal compartment syndrome after hip arthroscopy. Arthroscopy. 2010;26:128-30.

6. Ekman EF, Poehling GG. An experimental assessment of the risk of compartment syndrome during knee arthroscopy. Arthroscopy. 1996;12:193-9.

7. Belanger M, Fadale P. Compartment syndrome of the leg after arthroscopic examination of a tibial plateau fracture: case report and review of the literature. Arthroscopy. 1997;13:646-51.

8. Marti CB, Jakob RP. Accumulation of irrigation fluid in the calf as a complication during high tibial osteotomy combined with simultaneous arthroscopic anterior cruciate ligament reconstruction. Arthroscopy. 1999;15:864-6.

9. Arthroscopic irrigation/distention systems. Health Devices. 1999;28:242-81.

10. Ogilvie-Harris DJ, Weisleder L. Fluid pump systems for arthroscopy: a comparison of pressure control versus pressure and flow control. Arthroscopy. 1995;11:591-5. 